M. D. Voss $\cdot$ A. Beha $\cdot$ N. Tennagels $\cdot$ G. Tschank

A. W. Herling • M. Quint • M. Gerl •

C. Metz-Weidmann · G. Haun · M. Korn

\title{
Gene expression profiling in skeletal muscle of Zucker diabetic fatty rats: implications for a role of stearoyl-CoA desaturase 1 in insulin resistance
}

Received: 6 January 2005 / Accepted: 12 July 2005 / Published online: 12 November 2005

(C) Springer-Verlag 2005

\begin{abstract}
Aims/hypothesis: Insulin resistance in skeletal muscle is a hallmark of type 2 diabetes. Therefore, we sought to identify and validate genes involved in the development of insulin resistance in skeletal muscle. Materials: Differentially regulated genes in skeletal muscle of male obese insulin-resistant, and lean insulin-sensitive Zucker diabetic fatty (ZDF) rats were determined using Affymetrix microarrays. Based on these data, various aspects of glucose disposal, insulin signalling and fatty acid composition were analysed in a muscle cell line overexpressing stearoyl-CoA desaturase 1 (SCD1). Results: Gene expression profiling in insulin-resistant skeletal muscle revealed the most pronounced changes in gene expression for genes involved in lipid metabolism. Among these, $S c d 1$ showed increased expression in insulin-resistant animals, correlating with increased amounts of palmitoleoyl-CoA. This was further investigated in a muscle cell line that overexpressed SCD1 and accumulated lipids, revealing impairments of glucose uptake and of different steps of the insulin signalling cascade. We also observed differential effects of high-
\end{abstract}

\footnotetext{
Electronic supplementary material Supplementary material is available for this article at http://dx.doi.org/10.1007/s00125-0050025-2 and accessible for authorised users.
}

M. D. Voss $(\bowtie) \cdot$ A. Beha $\cdot$ N. Tennagels $\cdot$ G. Tschank

A. W. Herling - M. Korn

Sanofi-Aventis Deutschland GmbH,

Science and Medical Affairs, TD Metabolism,

Industriepark Hoechst,

65926 Frankfurt am Main, Germany

e-mail: MarcDietrich.Voss@sanofi-aventis.com

Tel.: +49-69-30516344

Fax: +49-69-30581901

M. Quint · M. Gerl

Sanofi-Aventis Deutschland GmbH, LO Biomarkers,

Frankfurt am Main, Germany

C. Metz-Weidmann · G. Haun

Sanofi-Aventis Deutschland GmbH, Genomic Sciences,

Frankfurt am Main, Germany glucose and fatty acid treatment on glucose uptake and long-chain fatty acyl-CoA profiles, and in particular an accumulation of palmitoleoyl-CoA in cells overexpressing SCD1. Conclusions/interpretation: Insulin-resistant skeletal muscle of ZDF rats is characterised by a specific gene expression profile with increased levels of Scd1. An insulinresistant phenotype similar to that obtained by treatment with palmitate and high glucose can be induced in vitro by overexpression of SCD1 in muscle cells. This supports the hypothesis that elevated SCD1 expression is a possible cause of insulin resistance and type 2 diabetes.

Keywords Affymetrix - Diabetes - Insulin resistance · SCD1 - Skeletal muscle - Stearoyl-CoA desaturase ZDF rat

Abbreviations ESM: Electronic Supplementary Material · GMI: Genetic Models Incorporated - HA: haemagglutin - LCACoA: long chain acyl-CoA - PTPN1: protein-tyrosine phosphatase $\mathrm{b} \cdot \mathrm{qRT}-\mathrm{PCR}$ : quantitative RT-PCR - SCD1: stearoyl-CoA desaturase $1 \cdot$ WT: wild-type

\section{Introduction}

Skeletal muscle is the major site of glucose utilisation and peripheral insulin action in response to nutritional challenges. It has long been known that dysregulation of fatty acid and lipid metabolism influences insulin signalling on various levels, leading to impaired glucose tolerance, decreased fatty acid oxidation and glycogen synthesis, and eventually resulting in insulin resistance and type 2 diabetes (reviewed in $[1,2]$ ). In particular, increased lipid accumulation in muscle, determined as intramyocellular lipid content, is frequently associated with insulin resistance [3-6]. Recently, it has also been implied that the degree of saturation of body lipids affects metabolic actions such as insulin signalling [7]. Stearoyl-CoA desaturase-1 (SCD1) is involved in the biosynthesis of mono-unsaturated fatty acids and catalyses the introduction of a double-bond at the 
$\Delta 9$ position of stearoyl (C18:0) and palmitoyl-CoA (C16:0). The products of SCD1 activity, oleic (C18:1 N9) and palmitoleic acids (C16:1 N7), constitute the majority of the mono-unsaturated fatty acids found in phospholipids, cholesterol esters and triglycerides. SCD1 expression is triggered by sterol regulatory element binding protein $1 \mathrm{c}$ [8] and is therefore highly regulated by a variety of stimuli, including leptin [9], insulin [10], glucose [11], cholesterol [12], iron [13], peroxisome proliferators [14] and polyunsaturated fatty acids [15].

Male Zucker diabetic fatty (ZDF) rats are a wellestablished animal model of insulin resistance and type 2 diabetes. Although experiments addressing changes in the transcriptional regulation of distinct genes in the development of insulin resistance or upon different treatments have been published $[16,17]$, to our knowledge a genome-wide, tissue-specific scan of differentially expressed genes in skeletal muscle of young ZDF rats has not yet been reported. Here, we describe a genome-scale Affymetrix gene expression study of genes differentially expressed in skeletal muscle of male ZDF rats with developing insulin resistance and type 2 diabetes compared with lean insulinsensitive controls. Since we observed highly elevated $S c d 1$ expression in insulin-resistant and diabetic muscle of the obese ZDF rats, the impact of SCD1 on insulin signalling and glucose disposal in skeletal muscle was further investigated in a cellular model.

\section{Subjects, materials and methods}

\section{Reagents}

Chemicals were purchased from Sigma (Deisenhofen, Germany) and cell culture reagents from Invitrogen (Karlsruhe, Germany). Rat anti-haemagglutin (HA) 3F10 and anti-MYC 9E10 antibodies were purchased from Roche (Mannheim, Germany), rabbit anti-(phospho-)AKT and anti-IRS1 antibodies from Biosource (Solingen, Germany) or Upstate/Biomol (Hamburg, Germany), antiphosphotyrosine antibody was from Santa Cruz (Heidelberg, Germany), anti-protein-tyrosine phosphatase b (PTPN1) antibody from Merck Bioscience (Schwalbach, Germany) and anti-beta-actin antibody from Sigma. Horseradish peroxidase-coupled secondary antibodies were from Amersham (Freiburg, Germany), Alexa Fluor 488-labelled anti-rat secondary antibody was from Molecular Probes (Karlsruhe, Germany).

\section{Animals and sample preparation}

Age-matched groups of obese male ZDF rats (Genetic Models Incorporated [GMI] $f a / f a$ ) and their lean littermates $(\mathrm{GMI}+/$ ?) were purchased from Charles River (Sulzfeld, Germany). The rats were housed in pairs at $20^{\circ} \mathrm{C}$ on a $12-\mathrm{h}$ light/12-h dark cycle with free access to water and standard rat diet (ssniff R/M-H; ssniff Spezialdiaeten, Soest, Germany) for 1 week upon arrival to allow recovery from transport. All experimental procedures were conducted according to the German Animal Protection Law. After $2 \mathrm{~h}$ of starvation ( $24 \mathrm{~h}$ for the group studied at 8 weeks of age), blood samples were drawn under terminal isoflurane. Tissue probes were excised rapidly and snap-frozen in liquid nitrogen. Determination of blood metabolic parameters was performed as described previously [18].

\section{Affymetrix microarray analysis}

Skeletal muscle probes (gluteus maximus, $150 \mathrm{mg}$ ) were lysed in RLT buffer (Qiagen, Hilden, Germany) with a homogeniser (UtraTurrax; Janke and Kunkel IKA Labortechnik, Staufen, Germany). Total RNA from the tissue lysates was isolated with the Qiagen RNeasy kit, including proteinase $\mathrm{K}$ digestion, DNase digestion and an additional RNeasy cleanup step, as recommended by the manufacturer. First- and second-strand cDNA syntheses were performed with $10 \mu \mathrm{g}$ of each total RNA using SuperScript SSII RT polymerase system (Invitrogen) and a T7(dT) ${ }_{24}$ primer. Double strand cDNA was extracted with phenolchloroform then precipitated with ethanol. Biotin-UPT- and -CTP-labelled cRNA was transcribed in vitro using the Enzo BioArray High Yield RNA Transcript Labeling Kit (Enzo Diagnostics, Farmingdale, NY, USA) and purified by RNeasy cleanup and ethanol precipitation. Aliquots of each total RNA and cRNA were monitored before and after the purification steps by UV spectrophotometry, agarose gel electrophoresis and BioAnalyzer RNA chips (Agilent, Boeblingen, Germany). cRNA samples $(15 \mu \mathrm{g})$ were fragmented at $94^{\circ} \mathrm{C}$ for $35 \mathrm{~min}$, added to hybridisation buffer and hybridised to RG-U34 A, B and C microarrays (Affymetrix, High Wycombe, UK) for $16 \mathrm{~h}$ at $45^{\circ} \mathrm{C}$ and $60 \mathrm{rev} / \mathrm{min}$. Microarrays were washed and double-stained with streptavidin-phycoerythrin conjugate (Molecular Probes) and anti-streptavidin antibody, using methods described by Affymetrix. After washing, the microarrays were analysed in a confocal GeneArray Scanner (HP, Boeblingen, Germany) with Microarray Suite Version 4.0 software (Affymetrix). Quality control of each chip was performed according to the Affymetrix quality criteria, including mean average difference, raw intensity and $3^{\prime} / 5^{\prime}$ ratio of the housekeeping genes beta-actin and glyceraldehyde-3-phosphate dehydrogenase. Expression profiling data were analysed using the in-house software GECKO 2 [19]. Briefly, this software performed a global normalisation on all microarrays using a reference chip for each group of biological replicates and the 75th percentile of the median of all transcribed genes. Biological replicates were subsequently agglomerated and a new matrix containing ratios and $p$-values was generated by crosswise element-by-element concatenation of all chips in one agegroup, and significance was assessed with Student's $t$-test. Merging of the respective biological replicates of each age (6,7 and 12 weeks) and phenotypic group (lean and obese) and subsequent filtering for $p$-values lower than 0.05 and changes in expression levels between the lean and obese groups higher than twofold resulted in the sets 
of differentially expressed genes reported here. The procedure we describe and the choice of a ratio of changes of at least twofold have been shown to ensure limitation of false positives and good reproducibility by alternative methods, e.g. quantitative RT-PCR (qRT-PCR) and Northern blotting $[19,20]$.

\section{Quantitative real-time PCR}

Total RNA $(1 \mu \mathrm{g})$ was reverse-transcribed with the AMVRT first-strand cDNA synthesis kit (Roche) in a $20 \mu \mathrm{l}$ reaction volume. Reverse-transcribed single strand cDNA $(2 \mu \mathrm{l})$ was used as a template for amplification in a Lightcycler using FastStart DNA Master Sybr Green according to the instructions of the manufacturer (Roche). Primers used were 5'-TGCTATGTTATCCTTTCTCTTG-3' and 5'-GGACACTTAATACACGATGTT G-3' (rat SCD1), 5'-CTTCTTCATCTTCACCTTCTTA-3', 5'-GCCCTAAGT ATTCAAGTTCTGT-3' (rat solute carrier family 2, member 4 [SLC2A4, also known as glucose transporter 4, GLUT4]), 5'-AAGTCCCTCACCCTCCCAAAAG-3' and 5'-CCTC AACACCTCAAACCACTCC-3' (rat beta-actin). The correct sizes of the resulting fragments $(121,136$ and 268 base pairs, respectively) were monitored by agarose gel electrophoresis. Total RNA contents were calculated using a concentration standard curve of the respective amplified fragments and normalised to expression levels of the housekeeping gene beta-actin. Confidence of the microarray data was assessed by TaqMan qRT-PCR of selected genes (covering a range of fold changes between +10.63 and -2.19 ), using the Applera TaqMan Universal PCR Master Mix and Pre-Developed TaqMan Assay Reagents on an ABI Prism 7900 device according to the instructions of the manufacturer (Applera, Norwalk, CT, USA). Endogenous RNA control 18S rRNA (Applera; 4310893E) was used for normalisation as an internal control. Table S1 of the Electronic Supplementary Material (ESM) summarises the design of primer and probe sequences and sequence identifiers of the commercially available primer and probe sets from Applera.

HPLC analysis of long-chain acyl-CoA species

HPLC analysis from freshly prepared $\mathrm{KH}_{2} \mathrm{PO}_{4}$ cell lysates was performed using a Waters Alliance 2690 system (2487 detector; Millennium 2010 chromatographic manager; Waters, Eschborn, Germany) as described elsewhere [18].

\section{Cloning and plasmids}

The complete open reading frame of human SCD1 was amplified from human skeletal muscle cDNA (Invitrogen) by PCR using primers 5'-CGGGATCCCGCCACCAT GCCGGCCCACTTGCTGCAG-3' and 5'-CCGCTCGA GCGGTCAAGCGTAGTCTGGGACGTCGTATGGGTA CATGCCACTCTTGTAGTTTCC-3'. The 3' primer introduced a sequence encoding a carboxy-terminal in-frame
HA epitope tag. The resulting fragment and pcDNA3.1(+) hygro vector (Invitrogen) were digested with $\mathrm{BamHI} / \mathrm{XhoI}$ and ligated to obtain pcDNA3.1 $(+)$ hygro-SCD1-HA. The identity of the construct was confirmed by sequencing.

Cell culture, transfections and immunofluorescence

Rat L6GLUT4myc myoblasts expressing MYC epitopetagged SLC2A4 (GLUT4) [21] were kindly provided by A. Klip (Hospital for Sick Children, Toronto, ON, Canada). Cells were maintained in alpha-MEM supplemented with $10 \%$ FCS, antibiotics (penicillin-streptomycin) and $2 \mu \mathrm{g} / \mathrm{ml}$ blasticidin at $37^{\circ} \mathrm{C}$ in $5 \% \mathrm{CO}_{2}$ and $95 \%$ humidity and were subcultured twice weekly. Transfections were carried out with L6GLUT4myc cells grown in six-well plates to $60 \%$ confluence, $2.5 \mu \mathrm{g}$ plasmid DNA and Fugene 6 reagent (Roche), as recommended by the manufacturer. For all transient transfections and for stably selected cell clones, empty pcDNA3.1(+) hygro vector served as negative control (subsequently referred to as 'wild-type' [WT]). Stable single-cell clones were selected with $500 \mu \mathrm{g} / \mathrm{ml}$ hygromycin B, picked manually and expanded. SCD1-HA expression levels were determined by SDS-PAGE and Western blotting (NuPAGE; Invitrogen) using anti-HA 3F10 monoclonal (Roche), anti-rat horseradish peroxidase secondary antibodies and chemiluminescence detection (Amersham). For fatty acid treatment, palmitate was dissolved in ethanol at $70^{\circ} \mathrm{C}$ to obtain a $20 \mathrm{mmol} / \mathrm{l}$ stock solution and coupled at a concentration of $200 \mu \mathrm{mol} / 1$ to $0.75 \%(\mathrm{w} / \mathrm{v})$ fatty-acid free BSA in cell culture medium for $1 \mathrm{~h}$ at $37^{\circ} \mathrm{C}$ to obtain a molar ratio of $2: 1$. Immunofluorescence assays were performed as described [22], using stable L6GLUT4myc SCD1-HA cells or L6GLUT4myc cells transiently transfected with pcDNA3.1(+) hygro SCD1-HA grown on coverslips to $70 \%$ confluence. SCD1-HA expression was detected using anti-HA 3F10 antibody (Roche) and anti-rat Alexa Fluor 488 antibody (Molecular Probes). Neutral lipid staining was performed with $1 \mu \mathrm{g} / \mathrm{ml}$ Nile Red (Sigma) in PBS for 10 min after fixation and permeabilisation of the cells. Nuclei were visualised using $1 \mu \mathrm{mol} / 1$ ToPro iodide in PBS (Molecular Probes). Confocal images were taken with a Leica TCS SP2 confocal laser scanning microscope.

2-Deoxyglucose uptake assay and analysis of insulin signalling

Stably transfected L6GLUT4myc cells were plated in 96well Cytostar-T scintillating microplates (Amersham) at $3.0 \times 10^{4}$ viable cells per well. After $32 \mathrm{~h}$ the cells were serum-starved with alpha-MEM supplemented with $2 \%$ newborn bovine serum and antibiotics (penicillin-streptomycin) for $16 \mathrm{~h}$. To analyse glucose uptake, the cells were washed twice in KRB, pH 7.3 (KRB), and incubated for 25 min with the given insulin concentrations in KRB. $\left[{ }^{14} \mathrm{C}\right]$ 2-deoxyglucose (0.01 MBq per well; Amersham) was added and the cells were incubated for another $25 \mathrm{~min}$ in a total 
volume of $150 \mu \mathrm{l}$ per well. Reactions were stopped by adding $10 \mu \mathrm{mol} / 1$ cytochalasin $\mathrm{B}$; plates were sealed and scintillation was measured in a Wallac Microbeta counter (Perkin Elmer, Rodgau-Juegesheim, Germany). Unspecific uptake was determined by incubating control wells with $20 \mu \mathrm{mol} / \mathrm{l}$ cytochalasin B and subtracted from each value. Insulin signalling was monitored in stably transfected L6GLUT4myc cells plated in 12-well plates upon direct stimulation with $10 \mathrm{nmol} / \mathrm{l}$ insulin for $15 \mathrm{~min}$ and subsequent lysis in 1 -TBS, $1 \% \mathrm{NP}-40,2 \mathrm{mmol} / 1$ sodium vanadate, $2 \mathrm{mmol} / \mathrm{l}$ Pefabloc and $1 \mu \mathrm{g} / \mathrm{ml}$ leupeptin. Levels of IRS1 tyrosine phosphorylation were quantified after immunoprecipitation of the proteins with anti-IRS1 antibody (Cell Signaling, Frankfurt, Germany) and immunoblotting using a phosphotyrosine antibody (Santa Cruz) and anti-IRS1 antibody for normalisation. AKT1 phosphorylation levels were determined with anti-phospho- $\mathrm{S}^{473}$ AKT1 (Biosource) and anti-AKT1 (Upstate) antibodies and direct immunoblotting of the lysates. Protein concentrations were determined with BCA protein assay (Pierce, Bonn, Germany).

\section{Statistical analysis}

Data are means \pm SEM of at least three independent experiments or show a representative graph of several independently performed experiments with similar results, as appropriate. Statistical significance was determined with Student's $t$-test ( $p$-values below 0.05 were considered significant) using the SigmaStat 2.03 program (SPSS, Chicago, IL, USA). Quantitative RT-PCR gene expression and phosphorylation data were calculated as fold change compared with controls and are given in arbitrary units (mean set to 1.0).

\section{Results}

Skeletal muscle-specific gene expression profiles of insulin-resistant ZDF rats

To investigate early tissue-specific events in the development of insulin resistance in skeletal muscle, we examined 34 obese male ZDF (GMI $f a / f a$ ) rats and lean controls $(\mathrm{GMI}+/$ ?) of three ages $(6,7$ and 12 weeks; $n=5-6)$. All obese ZDF rats exhibited the expected phenotype of in- creased weight gain and altered metabolic parameters (Table 1) compared with the respective age-matched controls. The 6- and 7-week-old obese ZDF rats showed the typical features of insulin resistance, such as hyperinsulinaemia (increased by 615 and $607 \%$ for the two ages, respectively; $p<0.01$ ) and elevated triglyceride levels (increased by 190 and $282 \% ; p=0.04$ ), but were normoglycaemic. The 12-week-old obese animals were already diabetic with hyperinsulinaemia (increased by $527 \%$; $p=0.04$ ), hyperglycaemia (glucose and $\mathrm{HbA}_{1} \mathrm{c}$ increased by 288 and $169 \%$, respectively; $p=0.02$ and $p=0.04$, respectively) and elevated triglyceride levels (increased by $639 \% ; p=0.006$ ).

To identify differentially expressed genes in skeletal muscle of these animals, we performed Affymetrix gene expression profiling using total RNA from gluteus maximus muscle and RGU-34A, B and C microarrays, covering in total 26,256 transcripts. An average of 3,724 \pm 69 (lean) and $3,701 \pm 65$ (obese) transcripts were expressed in muscle (3,621 vs. 3,605 [lean vs. obese, 6 weeks]; 3,793 vs. 3,790 [7 weeks]; 3,757 vs. 3,707 [12 weeks]). The total number of differentially expressed genes was 119 transcripts for the comparison between lean and obese ZDF rats in the 6-, 7and 12-week age groups. A complete list of the differentially expressed genes in the respective age groups is presented in the ESM (Tables S2-S4). Among the differentially expressed genes, there was a prevalence of genes involved in lipid metabolism, all of which were expressed more highly in insulin-resistant muscle (e.g. Cd36 [previously known as Fat], 2,4-dienoyl-CoA reductase, carnitine $o$-palmitoyltransferase, lipoprotein lipase, carnitine $o$-octanoyltransferase and stearoyl-CoA desaturase 1).

To assess the quality of the microarray data, a subset of eight genes was selected and expression levels were determined by TaqMan qRT-PCR. Higher expression in the obese animals of the respective age groups could be confirmed by qRT-PCR analysis for all the lipid metabolism genes mentioned above (ESM Table S5a-c). In addition to the time-points reported for the microarray analyses, minimally but significantly elevated expression levels in the obese animals could be detected by qRT-PCR for dienoyl-CoA reductase (weeks 6 and 7), carnitine octanoyltransferase (week 7) and lipoprotein lipase (week 7). However, only carnitine octanoyltransferase expression in week 7 (2.39-fold higher) exceeded a twofold change, whereas in the microarray experiments this was only

Table 1 Relevant metabolic parameters and body weights of male ZDF rats

\begin{tabular}{lcccccc}
\hline & $\begin{array}{l}\text { GMI+/? } \\
6 \text { weeks }\end{array}$ & $\begin{array}{l}\text { GMI } f a / f a \\
6 \text { weeks }\end{array}$ & $\begin{array}{l}\text { GMI+/? } \\
\text { 7 weeks }\end{array}$ & $\begin{array}{l}\text { GMI } f a / f a \\
7 \text { weeks }\end{array}$ & $\begin{array}{l}\text { GMI+/? } \\
12 \text { weeks }\end{array}$ & $\begin{array}{l}\text { GMI } f a / f a \\
12 \text { weeks }\end{array}$ \\
\hline Insulin $(\mathrm{ng} / \mathrm{ml})$ & $0.80 \pm 0.03$ & $4.92 \pm 0.30$ & $1.16 \pm 0,20$ & $7.05 \pm 0.54$ & $0.70 \pm 0.04$ & $3.69 \pm 0.35$ \\
$\mathrm{HbA}_{1} \mathrm{c}(\%)$ & $4.49 \pm 0.02$ & $4.54 \pm 0.07$ & $4.43 \pm 0.07$ & $4.46 \pm 0.05$ & $4.67 \pm 0.06$ & $7.88 \pm 1.56$ \\
Glucose (mmol/l) & $6.02 \pm 0.10$ & $7.62 \pm 0.42$ & $7.08 \pm 0.37$ & $6.93 \pm 0.19$ & $8.34 \pm 0.71$ & $24.06 \pm 3.10$ \\
Triglycerides (mmol/l) & $0.72 \pm 0.06$ & $1.37 \pm 0.12$ & $0.58 \pm 0.02$ & $1.64 \pm 0.19$ & $0.92 \pm 0.08$ & $5.88 \pm 0.84$ \\
Body weight (g) & $168.1 \pm 1.9$ & $198.4 \pm 2.8$ & $225.8 \pm 3.5$ & $281.9 \pm 5.0$ & $330.8 \pm 7.0$ & $410.2 \pm 7.1$ \\
Leptin (ng/ml) & $0.67 \pm 0.05$ & $21.03 \pm 2.17$ & $1.15 \pm 0.19$ & $39.95 \pm 2.00$ & $2.29 \pm 0.09$ & $23.12 \pm 1.25$ \\
\hline
\end{tabular}

Values are mean \pm SEM $(p<0.05 ; n=5-6)$ 


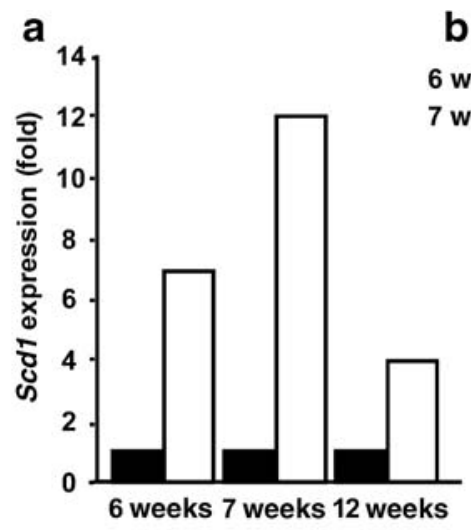

b

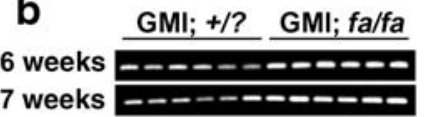

C

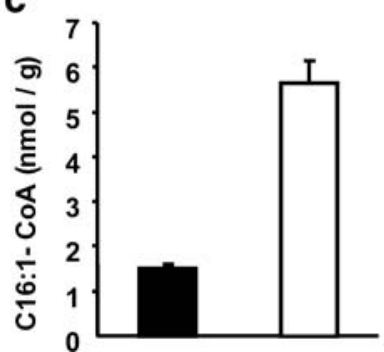

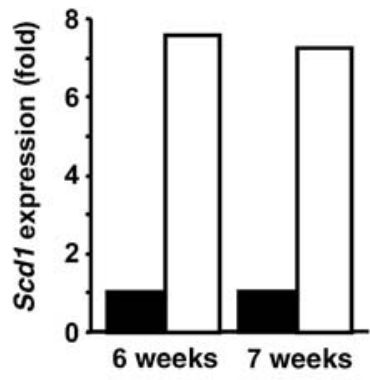

d

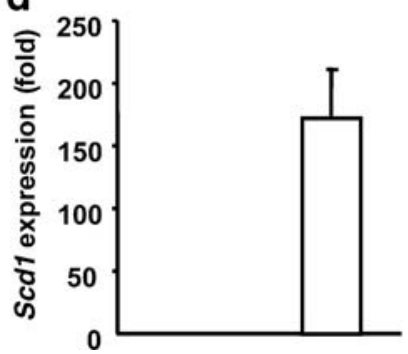

Fig. 1 Scd1 expression and C16:1 N7 palmitoleoyl-CoA content in ZDF rat skeletal muscle. a, b Relative expression levels of $S c d 1$ (a) on Affymetrix RG-U34A cDNA microarrays (qualifier J02585) and (b) in quantitative real-time PCR (upper panel integrity of bands in agarose gel electrophoresis; lower panel quantitation of Lightcycler data, normalised for beta-actin expression). Data are fold change vs controls (arbitrary units; $n=5-6 ; p<0.05$ ). c C16:1 N7 palmitoleoylCoA content in HPLC analysis of skeletal muscle from 8-week-old obese ZDF rats $(n=8 ; p<0.05)$. d Relative expression levels of rat $S c d 1$ in quantitative real-time PCR of skeletal muscle from 8-weekold obese ZDF rats. Data are fold change vs controls (arbitrary units; $n=8 ; p<0.05$; normalised for beta-actin expression). Filled columns, lean insulin-sensitive GMI+/? animals; open columns, obese insulinresistant GMI falfa animals

observed in week 12 . The decreased expression of only one gene, creatine kinase in week 12 , could not be reproduced by qRT-PCR: expression values for creatine kinase were extremely high in all groups and not regulated in the obese animals. In contrast, the opposite differential expression of metallothionein 2 between week 7 and 12 observed in microarrays could be confirmed by qRT-PCR.

Increased SCD1 expression and palmitoleoyl-CoA levels in insulin-resistant ZDF rats

Scd1 and $C d 36$ were the only genes that were consistently highly expressed in both Affymetrix and TaqMan qRTPCR analyses in all obese insulin-resistant or diabetic muscle probes tested (the increase in SCD1 expression was 5.9-, 10.6- and 3.2-fold in the 6-, 7- and 12-week-old animals, respectively; $p<0.01$; Fig. 1a). This prompted us to further investigate the role of SCD1 in the pathogenesis of insulin resistance. For example, elevated $S c d 1$ expression was further shown for the 6- and 7-week-old groups by Lightcycler quantitative real-time PCR using Scd1specific primers, and normalising for beta-actin expression a
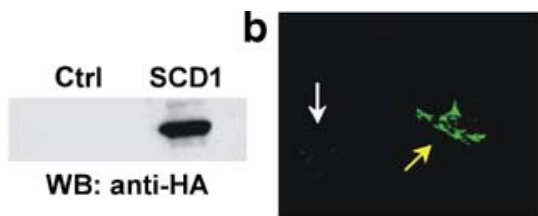

SCD1

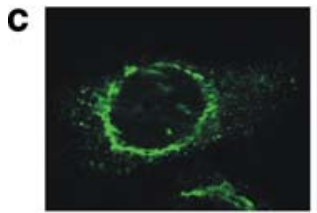

SCD1

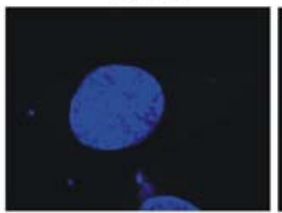

Nuclei

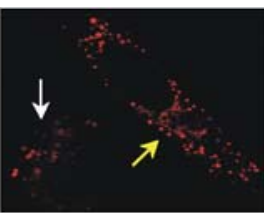

Nile red

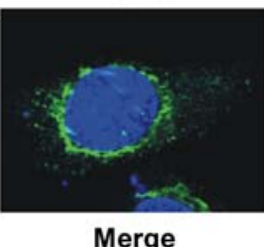

Fig. 2 SCD1 expression in myoblasts. a Expression of SCD1-HA in immunoblot analysis ( $W B$ Western blot) of transfected L6GLUT4myc myoblasts using anti-HA antibody 3F10 (Ctrl untransfected controls). b Confocal laser scanning analysis of neutral lipid accumulation in L6GLUT4myc myoblasts transiently overexpressing SCD1-HA (yellow arrow) or untransfected controls (white arrow). Cells were treated for $4 \mathrm{~h}$ with $200 \mu \mathrm{mol} / 1$ palmitate, neutral lipids were stained with $2 \mu \mathrm{g} / \mathrm{ml}$ Nile red (right panel) and SCD1 expression was immunostained with anti-HA antibody 3F10 (left panel). c Subcellular localisation of SCD1-HA in confocal laser scanning analysis of transfected L6GLUT4myc myoblasts using anti-HA antibody 3F10. Nuclei were visualised using $1 \mu \mathrm{mol} / 1$ ToPro iodide in PBS

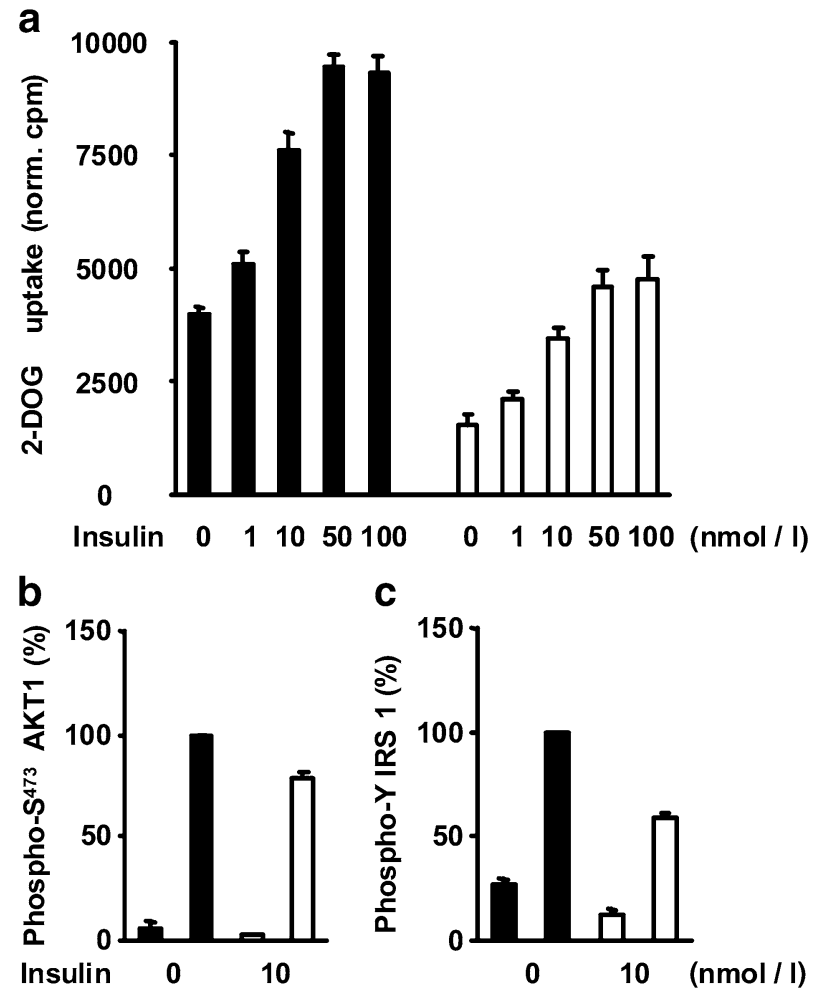

Fig. 3 Effect of SCD1 overexpression on basal and insulinstimulated glucose uptake and insulin signalling in myoblasts. a $\left[{ }^{14} \mathrm{C}\right]$ 2-deoxyglucose (2-DOG) uptake assay in stable SCD1 (open columns) and stably vector-transfected wild-type (filled columns) L6GLUT4myc cell clones. Values are mean \pm SEM from five independent cell lines tested $(n=8$; given as cpm normalised for unspecific uptake). b, c Analysis of insulin-stimulated AKT1 (S473) phosphorylation and IRS1 tyrosine respectively in stable SCD1 (open columns) and wild-type (filled columns) L6GLUT4myc cell clones by immunoprecipitation (pY-IRS1) and immunoblotting. Values are mean \pm SEM ( $n=3$; given as percentage of controls) 
(7.55- and 7.25-fold increase; $p=0.02$ and $p=0.008$, respectively; Fig. 1b). In an independent set of experiments, we additionally found that skeletal muscle from 8week-old obese ZDF rats, compared with their lean littermates, contained 3.72-fold $(p<0.01)$ higher amounts of palmitoleoyl-CoA (C16:1 N7) in HPLC analysis (Fig. 1c). Furthermore, much higher Scdl expression levels $(173$-fold; $p<0.01)$ were observed by quantitative real-time PCR (Lightcycler; Fig. 1d).

\section{A cellular model of SCD1 function}

To directly analyse the impact of SCD1 on insulin signalling and glucose disposal in muscle cells, we generated L6GLUT4myc cells that stably overexpressed human HAtagged SCD1 (referred to as SCD1 cells), confirmed SCD1 expression by Western blot analysis (Fig. 2a), and found SCD1 to be localised in endoplasmic reticulum-like subcellular structures by confocal laser scanning microscopy (Fig. 2c). Furthermore, Nile Red staining after 4 h of treatment with $200 \mu \mathrm{mol} / \mathrm{l}$ palmitate showed increased accumulation of neutral lipids in L6GLUT4myc cells transiently overexpressing SCD1 (Fig. 2b). For all subsequent experiments, controls cells (WT cells), derived from L6GLUT4myc cells stably transfected with empty control vector, served as controls.
SCD1 overexpression impairs glucose uptake and insulin signalling in vitro

2-Deoxyglucose uptake assays revealed impaired basal and insulin-stimulated glucose uptake in SCD1 compared with WT cells (mean decrease in five cell clones tested, 2.3-fold; $p<0.05$; Fig. 3a). By immunoprecipitation and Western blotting, we further analysed insulin-stimulated IRS1 and AKT1/protein kinase B phosphorylation patterns. Compared with WT cells, SCD1 cells showed 43\% $(p<0.05)$ decreased tyrosine-phosphorylation of IRS1 (Fig. 3b) and $27 \%(p<0.05)$ decreased serine ${ }^{473}$ phosphorylation of AKT1/protein kinase $\mathrm{B}$, indicating a negative impact of SCD1 expression on key points in insulin signalling.

SCD1 overexpression alters cellular gene expression levels

Screening for expression levels of genes known to be involved in or to be modulating insulin signalling revealed a 1.45-fold increased expression of PTPN1 protein in SCD1 cells (Fig. 4a) in Western blotting analysis. Upon differentiation, Lightcycler quantitative real-time PCR revealed that SCD1 cells contained 1.7-fold decreased transcript levels of the glucose transporter Slc2a4/Glut4 (Fig. 4b), whereas the protein level of the stably transfected MYC-tagged SLC2A4 remained unaltered in control experiments probing MYC-tag expression in immunoblot analysis (not shown).
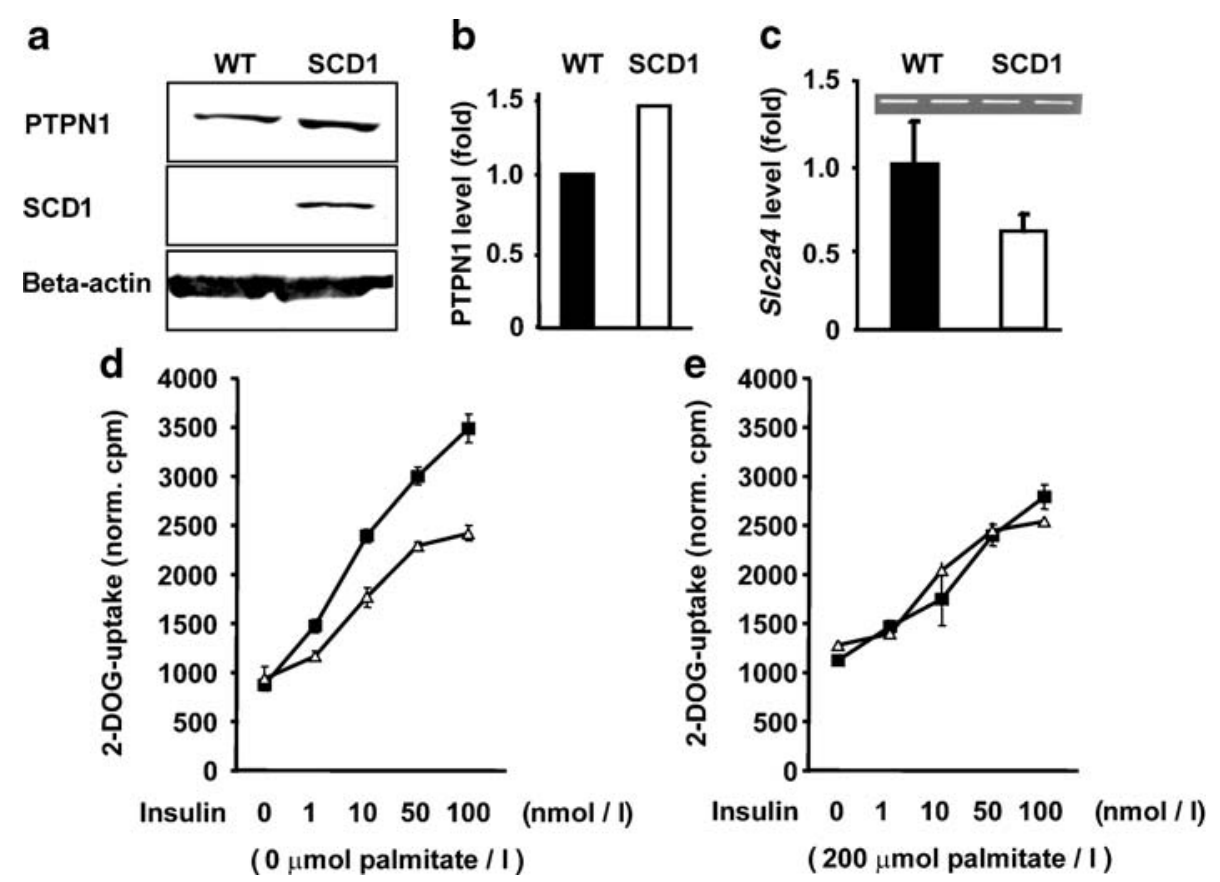

Fig. 4 Effect of SCD1 overexpression and palmitate treatment on insulin signalling in myoblasts. Immunoblot analysis (a) and quantitation (b) of PTPN1 protein expression in stable SCD1 and wild-type (WT) L6GLUT4myc cell clones using antibody FG6-1G. Data are fold change vs controls (arbitrary units). c Quantitative real-time PCR analysis of Slc2a4 (Glut4) transcript levels in fully differentiated stable SCD1 and wild-type L6GLUT4myc cell clones.
Values were normalised for beta-actin expression and are given as fold change vs controls (arbitrary units). d, e $\left[{ }^{14} \mathrm{C}\right] 2$-deoxyglucose (2DOG) uptake assay in stable SCD1 (open triangles) and wild-type (filled squares) L6GLUT4myc cell clones. Cells were incubated for $16 \mathrm{~h}$ at $20 \mathrm{mmol} / 1$ glucose without (d) or with (e) $200 \mu \mathrm{mol} / 1$ (right panel) palmitate. Values are given as cpm normalised for unspecific uptake. All panels show a representative experiment $(n=2)$ 
Table 2 HPLC analysis of amounts of C16 and C18 long-chain fatty acyl-CoA in WT and SCD1 myoblasts

\begin{tabular}{|c|c|c|c|c|}
\hline \multirow[t]{3}{*}{ LCACoA species } & \multicolumn{4}{|c|}{ Relative LCACoA amounts (nmol/ml) } \\
\hline & \multicolumn{2}{|l|}{ Control } & \multicolumn{2}{|l|}{ Treated } \\
\hline & WT & SCD1 & WT & SCD1 \\
\hline C 16:0 palmitoyl-CoA & $0.081 \pm 0.012$ & $0.061 \pm 0.001$ & $0.318 \pm 0.014$ & $0.444 \pm 0.024$ \\
\hline C 16:1 palmitoleoyl-CoA & $0.024 \pm 0.002$ & $0.167 \pm 0.020$ & $0.091 \pm 0.009$ & $0.177 \pm 0.002$ \\
\hline C 18:0 stearoyl-CoA & LOQ & LOQ & LOQ & LOQ \\
\hline C $18: 1$ oleoyl-CoA & $0.148 \pm 0.020$ & $0.222 \pm 0.009$ & $0.084 \pm 0.004$ & $0.178 \pm 0.031$ \\
\hline
\end{tabular}

Data were normalised for protein content and are given as means $\pm \mathrm{SD}(\mathrm{nmol} / \mathrm{ml}$ cell lysate; $n=2)$

Table shows a representative experiment

$L O Q$ Below limit of quantification, Treated incubated with palmitate $200 \mu \mathrm{mol} / \mathrm{l}$ for $16 \mathrm{~h}$

SCD1 overexpression alters cellular long-chain acyl-CoA levels

To assess the impact of SCD1 overexpression on highglucose- and fatty acid-induced insulin resistance, glucose uptake assays were performed upon treatment with high palmitate $(200 \mu \mathrm{mol} / \mathrm{l})$ and glucose $(20 \mathrm{mmol} / \mathrm{l})$. Glucose alone rendered SCD1 cells less insulin-sensitive, but did not alter basal glucose uptake (Fig. 4d). Additional treatment with $200 \mu \mathrm{mol} / 1$ palmitate decreased the insulin sensitivity of WT cells to the level of SCD1 cells (maximum insulin stimulation was reduced to 58\%), whereas the latter did not exhibit any further impairment of insulin sensitivity (Fig. 4e).

To investigate whether the observed phenotype regarding insulin sensitivity is reflected in the products of SCD1 enzymatic activity and alterations in long-chain fatty acylCoA levels, HPLC analyses from $\mathrm{KH}_{2} \mathrm{PO}_{4}$ lysates of palmitate-treated SCD1 and WT cells were performed. Table 2 demonstrates that C16:0 levels were slightly increased in WT compared with SCD1 cells, but highly elevated in both cell lines after palmitate treatment (393 and 727\%). C18:0 levels were below the detection limit in all probes tested, although more than $10^{7}$ cells were an-

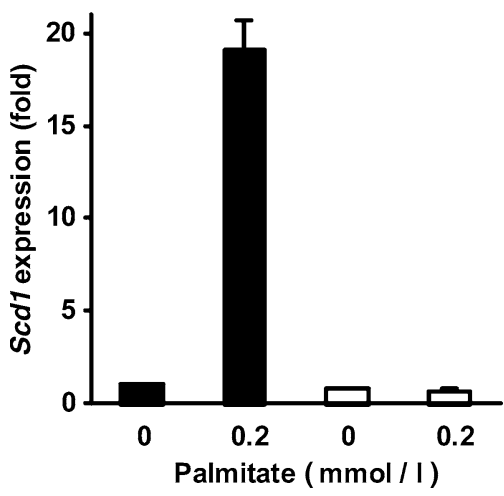

Fig. 5 Effect of palmitate treatment on endogenous $\mathrm{Scd} 1$ expression in SCD1 (open columns) and wild-type (filled columns) L6GLUT4myc myoblasts. Cells were incubated for $16 \mathrm{~h}$ at $20 \mathrm{mmol} / \mathrm{l}$ glucose with or without $200 \mu \mathrm{mol} / 1$ palmitate, as described for Fig. 4 and Table 2. Scdl expression levels were determined by quantitative real-time PCR. A representative experiment is shown, giving results as fold change vs controls ( $n=2$; arbitrary units, normalised for betaactin expression) alysed per data point. Treatment with palmitate at high glucose concentrations moderately diminished C18:1 N9 (176 and 125\%), but increased C16:1 N7 levels (379 and $110 \%)$ in both WT and SCD1 cells. Interestingly, SCD1 cells per se contained slightly elevated levels of C18:1 N9 (150\%) but significantly higher levels of C16:1 N7 (696\%) than WT cells. Quantitative RT-PCR analysis further revealed that palmitate treatment increased $S c d 1$ transcript levels in WT cells 19.1-fold (Fig. 5), but endogenous Scd1 levels in SCD1 cells remained mainly unaltered (1.13-fold decrease).

\section{Discussion}

Skeletal muscle is the major site of glucose disposal in the body. Here we report the first genome-wide, tissue-specific screen for genes differentially expressed in skeletal muscle of insulin-resistant and diabetic ZDF rats. We observed a unique gene expression profile with a prevalence of lipidmetabolising enzymes among the differentially expressed genes, which was confirmed by TaqMan qRT-PCR. The striking and consistent overexpression of $S c d 1$ in the obese animals of all ages tested supports the hypothesis that muscle reacts to a high fatty acid burden by regulating key enzymes in lipid metabolism, whereas most transcriptional changes probably occur in adipose tissue. Interestingly, in muscle of insulin-resistant animals (6 and 7 weeks of age), $S c d 1$ and $C d 36$ were initially increased, whereas dienoylCoA reductase, a protein involved in beta-oxidation, first became increased at a later time-point in animals with established diabetes (12 weeks of age). Similar changes towards elevated lipid utilisation in already diabetic ZDF rats have been observed in the kinetic studies of Etgen and Oldham [23]. Cd36 transgenic and knock-out mice models further revealed a correlation of insulin resistance, increased fatty acid influx and increased $C d 36$ expression in muscle $[24,25]$. However, in the qRT-PCR data slightly but significantly elevated dienoyl-CoA reductase expression was already observed in the 6- and 7-week-old insulinresistant animals, implying that the direction of the observed changes was already defined at this stage.

The second largest group of genes dysregulated in our experiments was involved in protein trafficking, catabolism and cytoskeleton integrity (e.g. the cytosolic sorting 
protein Pacs1, ubiquitin-conjugating enzyme E2-3, alphatropomyosin, slow skeletal muscle troponin, alpha-actin and beta-myosin), supporting the notion that changes in membrane and cytoskeleton composition accompany insulin resistance in muscle. Increased expression of metallothionein 2 in week 12 could represent a response against beginning oxidative stress in the already diabetic state. From the GECKO analysis, we estimated a false-positive rate of 5\%. Accordingly, we could not reproduce any differential expression of creatine kinase by qRT-PCR. As we observed extremely high expression levels of this mRNA in all probes, oversaturated signals could also have accounted for this discrepancy.

The prominent expression of Scdl that we report in insulin resistance is in agreement with other rodent models of obesity and insulin resistance, such as $o b / o b$ mice $[9,26$, 9]. The differences in the absolute levels of $S c d 1$ transcript overexpression in the different experiments reported for the 6-, 7- and 12-week-old (Fig. 1a,b) rats compared with the extremely high values for the 8-week-old ZDF rats (Fig. 1d) probably result from different times of starvation ( 2 vs. 24 h) before the analyses. Due to the high turnover and fast induction of SCD1, the transcript levels do not necessarily reflect SCD1 enzymatic activities or products in a linear manner.

In humans, an abundance of the SCD1 product C16:1 N7 palmitoleic acid has also been described in insulin resistance and obesity [7]. We further established a causative role for SCD1 in muscle insulin resistance in cell culture experiments: SCD1 protein stably overexpressed in recombinant muscle cells was functional regarding its subcellular localisation and increased neutral lipid accumulation upon palmitate treatment. Interestingly, these SCD1 cells exhibited impaired glucose uptake, decreased insulin sensitivity at various steps of the insulin signalling cascade and dysregulation of known key regulators of insulin action. This is consistent with and extends the phenotype of $S c d 1-/-$ mice [27, 28], as our data indicate that the improved insulin sensitivity observed for $S c d 1-/-$ mice is a direct result of the lack of $S c d 1$ expression rather than that it is caused by adaptive changes during development of the mice, or is a simple result of ameliorated obesity in $S c d 1-/-$ mice.

In addition to the knockout model, Houdali et al. triggered insulin resistance by prolonged glucose infusion into skeletal muscle of Wistar rats and correlated the resulting impairment in insulin signalling with increasing $S c d 1$ expression levels and activities in vivo [29, 30]. In our cell culture model, stable overexpression of SCD1 alone was sufficient to impair glucose uptake and insulin signalling, indicating that SCD1 levels or enzyme activity determined the observed phenotypic changes and thus supporting the findings of Houdali et al. Analysis of functional glucose uptake data and HPLC LCACoA profiles upon palmitate treatment of SCD1-overexpressing vs. WT cells revealed that palmitate treatment in high-glucose conditions could lower the insulin sensitivity of WT cells to the level of SCD1 cells per se. Additionally, this treatment increased C16:1 N7 levels and endogenous $S c d 1$ transcript levels in WT but not in SCD1 cells, which contained higher amounts of stably transfected SCD1. This implies that, in SCD1 cells, feedback mechanisms besides polyunsaturated fatty acids were limiting endogenous $S c d 1$ expression at the level of transcription-probably the mono-unsaturated SCD1 product 16:1 N7 itself [31]. However, the underlying mechanisms remain unclear. As all samples were analysed at $20 \mathrm{mmol} / \mathrm{l}$ glucose, we cannot define any further the extent to which glucose itself increased endogenous $S c d 1$ expression (e.g. compared with cells incubated at $5.5 \mathrm{mmol} / 1$ glucose), but we observed clear induction of $S c d 1$ when WT cells were incubated with both $200 \mu \mathrm{mol} / 1 \mathrm{palmitic}$ acid and $20 \mathrm{mmol} / \mathrm{l} \mathrm{high-glucose}$ solution.

Elevated LCACoAs levels have been proposed to account for insulin resistance $[32,33]$. However, our data suggest that LCACoA composition, in particular the relative proportion of mono-unsaturated $16: 1 \mathrm{~N} 7$, is probably the more important determinant. As our HPLC analysis addressed only changes in LCACoA, we cannot determine where C16:1 N7 mono-unsaturated fatty acids may further exert a negative impact on cellular insulin signalling: as a component of phospholipids on the level of membrane composition or as ceramide or di- or triglycerides, all of which are fractions known to be associated with insulin sensitivity and to have a composition known to be altered by SCD1 activity [31,34]. Our observations point to the existence of direct effects of SCD1, or the products of its enzymatic activity (in particular C16:1 N7), on immediate signalling events, and of indirect downstream effects on the level of transcription or protein expression. In particular, treatment of WT cells with high glucose and high palmitate mimicked the phenotype of SCD1 overexpression. As SCD1 is highly regulated by nutritional challenges, it is tempting to speculate that nutritional oversupply of glucose and saturated fatty acid species could trigger insulin resistance by upregulating $S c d 1$ expression, which in turn elevates C16:1 N7 levels. Further studies should elucidate the exact mechanisms underlying the negative effects of SCD1 and mono-unsaturated C16:1 N7 fatty acyl Co-A species on insulin signalling and (post-)transcriptional regulation.

Acknowledgements We are grateful to Dr Amira Klip (Hospital for Sick Children, Toronto, ON, Canada) for kindly providing L6 cells overexpressing GLUT4myc. We thank Gertraud Jerabek-Sandow for help with qRT-PCR, Stefan Welte for help with analysing IRS1 and AKT1 phosphorylation, and Dr Guenter Mueller for helpful discussions (all at Sanofi-Aventis, TD Metabolism, Frankfurt, Germany).

Duality of interest

All authors are employees of Sanofi-Aventis Deutschland GmbH, Frankfurt, Germany.

\section{References}

1. McGarry JD (2002) Banting lecture 2001: dysregulation of fatty acid metabolism in the etiology of type 2 diabetes. Diabetes 51:7-18 
2. Lewis GF, Carpentier A, Adeli K, Giacca A (2002) Disordered fat storage and mobilization in the pathogenesis of insulin resistance and type 2 diabetes. Endocr Rev 23:201-229

3. Virkamaki A, Korsheninnikova E, Seppala-Lindroos A et al (2001) Intramyocellular lipid is associated with resistance to in vivo insulin actions on glucose uptake, antilipolysis, and early insulin signaling pathways in human skeletal muscle. Diabetes 50:2337-2343

4. Boden G, Chen X, Capulong E, Mozzoli M et al (2001) Effects of free fatty acids on gluconeogenesis and autoregulation of glucose production in type 2 diabetes. Diabetes 50:810-816

5. Boden G, Jadali F, White J et al (1991) Effects of fat on insulinstimulated carbohydrate metabolism in normal men. J Clin Invest 88:960-966

6. Kuhlmann J, Neumann-Haefelin C, Belz U et al (2003) Intramyocellular lipid and insulin resistance: a longitudinal in vivo $1 \mathrm{H}$-spectroscopic study in Zucker diabetic fatty rats. Diabetes 52:138-144

7. Vessby B, Gustafsson IB, Tengblad S, Boberg M, Andersson A (2002) Desaturation and elongation of fatty acids and insulin action. Ann N Y Acad Sci 967:183-195

8. Shimomura I, Shimano H, Korn BS, Bashmakov Y, Horton JD (1998) Nuclear sterol regulatory element-binding proteins activate genes responsible for the entire program of unsaturated fatty acid biosynthesis in transgenic mouse liver. J Biol Chem 273:35299-35306

9. Cohen P, Miyazaki M, Socci ND, Hagge-Greenberg A et al (2002) Role for stearoyl-CoA desaturase-1 in leptin-mediated weight loss. Science 297:240-243

10. Frick F, Linden D, Ameen C, Eden S, Mode A, Oscarsson J (2002) Interaction between growth hormone and insulin in the regulation of lipoprotein metabolism in the rat. Am J Physiol Endocrinol Metab 283:1023-1031

11. Jones BH, Standridge MK, Claycombe KJ, Smith PJ, Moustaid-Moussa N (1998) Glucose induces expression of stearoyl-CoA desaturase in 3T3-L1 adipocytes. Biochem J 335: 405-408

12. Landau JM, Sekowski A, Hamm MW (1997) Dietary cholesterol and the activity of stearoyl CoA desaturase in rats: evidence for an indirect regulatory effect. Biochim Biophys Acta 1345:349-357

13. Falvella FS, Pascale RM, Gariboldi M et al (2002) StearoylCoA desaturase 1 (Scd1) gene overexpression is associated with genetic predisposition to hepatocarcinogenesis in mice and rats. Carcinogenesis 23:1933-1936

14. Miller CW, Ntambi JM (1996) Peroxisome proliferators induce mouse liver stearoyl-CoA desaturase 1 gene expression. Proc Natl Acad Sci U S A 93:9443-9448

15. Ntambi JM (1992) Dietary regulation of stearoyl-CoA desaturase 1 gene expression in mouse liver. J Biol Chem 267: 10925-10930

16. Pattison JS, Folk LC, Madsen RW, Booth FW (2003) Selected contribution: identification of differentially expressed genes between young and old rat soleus muscle during recovery from immobilization-induced atrophy. J Appl Physiol 95:2171-2179

17. Alon T, Friedman JM, Socci ND (2003) Cytokine-induced patterns of gene expression in skeletal muscle tissue. J Biol Chem 278:32324-32334
18. Neumann-Haefelin C, Beha A, Kuhlmann J, Belz U et al (2004) Muscle-type specific intramyocellular and hepatic lipid metabolism during starvation in Wistar rats. Diabetes 53:528-534

19. Theilhaber J, Ulyanov A, Malanthara A et al (2004) GECKO: a complete large-scale gene expression analysis platform. BMC Bioinformatics 5:195-209

20. Becker W, Kluge R, Kantner T et al (2004) Differential hepatic gene expression in a polygenic mouse model with insulin resistance and hyperglycemia: evidence for a combined transcriptional dysregulation of gluconeogenesis and fatty acid synthesis. J Mol Endocrinol 32:195-208

21. Wang Q, Khayat Z, Kishi K, Ebina Y, Klip A (1998) GLUT4 translocation by insulin in intact muscle cells: detection by a fast and quantitative assay. FEBS Lett 427:193-197

22. Voss MD, Hille A, Barth S, Spurk A et al (2001) Functional cooperation of Epstein-Barr virus nuclear antigen 2 and the survival motor neuron protein in transactivation of the viral LMP1 promoter. J Virol 75:11781-11790

23. Etgen GJ, Oldham BA (2000) Profiling of Zucker diabetic fatty rats in their progression to the overt diabetic state. Metabolism 49:684-688

24. Hajri T, Han XX, Bonen A, Abumrad NA (2002) Defective fatty acid uptake modulates insulin responsiveness and metabolic responses to diet in CD36-null mice. J Clin Invest 109: 1381-1389

25. Ibrahimi A, Bonen A, Blinn WD et al (1999) Muscle-specific overexpression of FAT/CD36 enhances fatty acid oxidation by contracting muscle, reduces plasma triglycerides and fatty acids, and increases plasma glucose and insulin. J Biol Chem 274:26761-26766

26. Enser M (1975) Desaturation of stearic acid by liver and adipose tissue from obese-hyperglycaemic mice $(o b / o b)$. Biochemistry J 148:551-555

27. Ntambi JM, Miyazaki M, Stoehr JP et al (2003) Loss of stearoyl-CoA desaturase-1 function protects mice against adiposity. Proc Natl Acad Sci U S A 99:11482-11486

28. Rahman SM, Dobrzyn A, Dobrzyn P, Lee SH, Miyazaki M, Ntambi JM (2003) Stearoyl-CoA desaturase 1 deficiency elevates insulin-signaling components and down-regulates proteintyrosine phosphatase 1B in muscle. Proc Natl Acad Sci U S A 100:11110-11115

29. Houdali B, Wahl HG, Kresi M et al (2003) Glucose oversupply increases Delta9-desaturase expression and its metabolites in rat skeletal muscle. Diabetologia 46:203-212

30. Houdali B, Nguyen V, Ammon HP, Haap M et al (2002) Prolonged glucose infusion into conscious rats inhibits early steps in insulin signalling and induces translocation of GLUT4 and protein kinase $C$ in skeletal muscle. Diabetologia 45:356368

31. Ntambi JM, Miyazaki M (2004) Regulation of stearoyl-CoA desaturases and role in metabolism. Prog Lipid Res 43:91-104

32. Cooney GJ, Thompson AL, Furler SM, Ye J, Kraegen EW (2002) Muscle long-chain acyl CoA esters and insulin resistance. Ann N Y Acad Sci 967:196-207

33. Hegarty BD, Furler SM, Ye J, Cooney GJ, Kraegen EW (2003) The role of intramuscular lipid in insulin resistance. Acta Physiol Scand 178:373-383

34. Ntambi JM, Miyazaki M (2003) Recent insights into stearoylCoA desaturase. Curr Opin Lipidol 14:255-261 